\title{
Magnetic Gradiometer Based on a High-Transition Temperature Superconducting Quantum Interference Device for Improved Sensitivity of a Biosensor
}

\author{
SeungKyun Lee, W. R. Myers, H. L. Grossman, Y. R. Chemla, and John Clarke \\ Department of Physics, University of California, Berkeley, CA 94720 and \\ Materials Sciences Division, Lawrence Berkeley National Laboratory, Berkeley, CA \\ 94720
}

We describe a gradiometer based on a high-transition temperature Superconducting Quantum Interference Device (SQUID) for improving the sensitivity of a SQUID-based biosensor. The first-derivative gradiometer, fabricated from a single layer of $\mathrm{YBa}_{2} \mathrm{Cu}_{3} \mathrm{O}_{7-\mathrm{x}}$, has a baseline of $480 \mu \mathrm{m}$ and a balance against uniform fields of 1 part in 150. Used in our SQUID "microscope," it reduces parasitic magnetic fields generated by the measurement process to the level of the SQUID noise. The gradiometer-based microscope is two orders of magnitude more sensitive to superparamagnetic nanoparticles bound to biological targets than our earlier magnetometer-based microscope.

85.25.Dq, 87.80.-y 
There is increasing interest in the use of Superconducting Quantum Interference Devices (SQUIDs) to monitor samples at room temperature ${ }^{1}$, especially for biological studies 2345 678 and nondestructive evaluation of materials ${ }^{9} 10^{11} 12$. In particular, SQUID "microscopes" are used as rapid, versatile and sensitive biosensors ${ }^{567}$ to detect superparamagnetic nanoparticles attached to pathogens or cells by means of an appropriate antibody-antigen interaction. In one such scheme ${ }^{5}$, the resolution was estimated to be approximately 50,000 particles. Since typically many particles are attached to a single biological target, the number of targets detected is likely to be substantially smaller, making this technique potentially competitive for biological assays. In these experiments, the resolution was limited not by the intrinsic noise of the hightransition temperature $\left(\mathrm{T}_{\mathrm{c}}\right)$ SQUID magnetometer but rather by parasitic magnetic fields generated by the magnetic pulses used in the detection scheme. In this letter we demonstrate that the contribution from these parasitic fields can be greatly reduced by replacing the SQUID magnetometer with a gradiometer ${ }^{13}$, thereby improving the sensitivity by a factor of 80 . High- $\mathrm{T}_{\mathrm{c}}$ gradiometers have also been shown to reduce noise from the external environment ${ }^{8}$.

The inset of Fig. 1 shows the configuration of the SQUID and sample in our microscope ${ }^{14}$. The thin film, $\mathrm{YBa}_{2} \mathrm{Cu}_{3} \mathrm{O}_{7-\mathrm{x}}(\mathrm{YBCO}) \mathrm{SQUID}$ is mounted on top of a sapphire rod which is thermally anchored to a can of liquid nitrogen; these components are enclosed in a fiberglass vacuum chamber. A $3 \mu \mathrm{m}$-thick, $410 \times 410 \mu \mathrm{m}^{2}$ silicon nitride window patterned in a $0.5 \mathrm{~mm}$-thick Si chip separates the SQUID from the sample well, which has a volume of about $0.3 \mu \mathrm{L}$. Using three micrometers to lower the 
window, we typically bring the sample to within $100 \mu \mathrm{m}$ of the SQUID. The entire system is operated inside a triple-layer cylindrical $\mu$-metal shield, which reduces the earth's field to about $4 \mathrm{nT}$ and eliminates ambient magnetic field fluctuations.

In our earlier experiments with a SQUID magnetometer ${ }^{5}$, we pulsed a magnetic field parallel to the plane of the magnetometer and detected the subsequent Néel relaxation of superparamagnetic nanoparticles. In the absence of any sample, we observed an exponentially decaying magnetic field with a time constant of about $30 \mathrm{~ms}$; an example is shown in Fig. 1. This decaying field limits the resolution. On investigation, we found that the decaying field originated in either eddy currents induced in metal components or reorientation of magnetic domains in the $\mu$-metal. To reduce the contribution from this parasitic field, we replaced the magnetometer with the gradiometer shown in Fig. 2. A uniform magnetic field $\mathrm{B}_{\mathrm{z}}$ induces a supercurrent around the rectangular washer, while a gradient $\partial \mathrm{B}_{\mathrm{Z}} / \partial \mathrm{y}$ generates a supercurrent in the center trace, coupling a flux

$$
\Phi_{\mathrm{s}} \approx \mathrm{A}_{\mathrm{s}} \mathrm{B}_{\mathrm{z}}+\alpha\left(\mathrm{L}_{\mathrm{s}} / \mathrm{L}_{\mathrm{p}}\right) \mathrm{A}_{\mathrm{p}}\left(\mathrm{B}_{\mathrm{z1}}-\mathrm{B}_{\mathrm{z} 2}\right)
$$

into the SQUID. Here, $\mathrm{A}_{\mathrm{s}}$ is the effective area of the SQUID, $\mathrm{L}_{\mathrm{s}}$ is its inductance, $\alpha$ is the fraction of the SQUID circumference through which supercurrent flows, $A_{p}$ is the effective area of each pickup loop (estimated as the geometric mean of the inner and outer areas), $L_{p}$ is the inductance of each loop including the contribution $\alpha L_{s}$ from the SQUID, $\mathrm{B}_{\mathrm{z}}$ is the average uniform magnetic field, and $\mathrm{B}_{\mathrm{zl}}$ and $\mathrm{B}_{\mathrm{z2}}$ are the average fields applied to the upper and lower loops in Fig. 2. 
The gradiometer was designed to achieve good coupling to the sample. We calculated the coupling by modeling the sample as a uniform distribution of magnetic dipoles, magnetized in plane, on the microscope window. When the gradiometer is centered beneath the sample, the flux $\Phi_{z 1}$ through one pickup loop is equal and opposite to the flux through the other pickup loop, $\Phi_{z 2}$, inducing a supercurrent $2 \Phi_{z 1} / L_{p}$ around the SQUID loop and a flux $2 \alpha \Phi_{z 1} L_{s} / L_{p}$ into the SQUID. Assuming the gradiometer to be positioned $100 \mu \mathrm{m}$ below the sample, we calculated the geometry of the pickup loops which would maximize the flux coupled into the SQUID and designed the gradiometer with approximately these dimensions ${ }^{15}$. The resulting gradiometer had an effective width of $400 \mu \mathrm{m}$ and a baseline of $480 \mu \mathrm{m}$, dimensions comparable to the sample size. We calculated ${ }^{16} \mathrm{~L}_{\mathrm{p}} \approx 630 \mathrm{pH}$, chose SQUID parameters such that $\mathrm{L}_{\mathrm{s}} \approx 55 \mathrm{pH}$ (the upper limit for good SQUID performance), and estimated $\alpha \approx 0.8$. The use of narrow $(4 \mu \mathrm{m})$ lines for the SQUID reduced flux entry ${ }^{17}$. Since the nearest metal objects are $50 \mathrm{~mm}$ away, we anticipated that the short-baseline gradiometer would substantially suppress the parasitic field.

To compare the coupling of the gradiometer with that of our magnetometer ${ }^{5}$, we modeled the magnetometer as a $128 \times 128 \mu \mathrm{m}^{2}$ square $(128 \mu \mathrm{m}$ being the geometric mean of the inner and outer dimensions of the washer), located $50 \mu \mathrm{m}$ beneath the sample and offset $205 \mu \mathrm{m}$ (half the sample length) from center. As with the gradiometer, we calculated the flux coupled in from a uniform distribution of dipoles. We estimated that gradiometer would have only 0.4 times as much flux coupled into it as the magnetometer. Despite 
this loss in coupling efficiency, we anticipated a net gain in resolution due to the expected reduction of the parasitic field contribution.

We fabricated gradiometers by laser-depositing a $200 \mathrm{~nm}$-thick YBCO film on a $30^{\circ}$ $\mathrm{SrTiO}_{3}$ bicrystal and patterning it with an ion mill. We made electrical contact to the SQUID leads by means of evaporated $\mathrm{Ag}$ traces insulated from the pickup loops by a layer of $\mathrm{SiO}$. These traces extended over the beveled chip edge; electrical contact to the wires was made with Ag paste. A multiturn gradiometric modulation/feedback coil was patterned in a Ag film evaporated onto a sapphire chip. We used Apiezon ${ }^{\circledR} \mathrm{N}$-grease to attach the gradiometer to this chip which, in turn, was glued to the sapphire rod. Operated in a flux-locked loop with bias reversal ${ }^{18}$, the SQUID achieved a flux noise of $17 \mu \Phi_{0} \mathrm{~Hz}^{-1 / 2}$ at frequencies down to $2 \mathrm{~Hz}$. The measured effective area of the gradiometer in response to a uniform field is $94 \mu \mathrm{m}^{2}{ }^{19}$; dividing this by the modeled effective area of one pickup loop, $\alpha \mathrm{A}_{\mathrm{p}}\left(\mathrm{L}_{\mathrm{s}} / \mathrm{L}_{\mathrm{p}}\right) \approx 14,000 \mu \mathrm{m}^{2}$, yields a balance of about 1 part in 150. Magnetization fields greater than $1.2 \mathrm{mT}$ induce flux jumping.

Figure 1 compares the response of the gradiometer to a $1.2 \mathrm{mT}$ field, pulsed on and off at $1 \mathrm{~s}$ intervals, with that of our earlier magnetometer with $0.3 \mathrm{mT}$ pulses. The amplitude of the parasitic field detected by the gradiometer has been reduced to a level comparable to the SQUID noise. As a result, the previous detection limit of $87 \mu \Phi_{0}$, due to the background decay detected by the magnetometer, has been reduced by a factor of 30 to $2.9 \mu \Phi_{0}$, the uncertainty in the fitted value of $\Phi_{\mathrm{s}}$ due to SQUID noise. 
To measure superparamagnetic particles, we positioned the microscope window 100610 $\mathrm{m}$ from the gradiometer. This distance was limited by the uncertainty in the angular misalignment between the SQUID chip and the window. To compare the coupling of a given sample to the gradiometer and magnetometer, we measured five samples, consisting of magnetic particles immobilized on a substrate, with each system. On average, the gradiometer signal was 0.8 times that of the magnetometer. This is about two times greater than our predicted value and illustrates the uncertainties in our estimates of the various parameters. The gradiometer signal could be increased by decreasing the SQUID-sample separation. However, calculations showed, and measurements confirmed, that the coupling between the gradiometer and sample depends only weakly on distance for distances smaller than the baseline (inset Fig. 3). Therefore, even if the sample-SQUID separation could be reduced to $50 \mathrm{~m}$, the separation achieved with the magnetometer, the gradiometer signal would increase by a factor of only 1.4.

A significant advantage of the gradiometer is that, by effectively eliminating the parasitic background field, a larger magnetic field can be applied to the sample without a concomitant increase in the background response. Therefore, we were able to apply a 4 times larger field, $1.2 \mathrm{mT}$, in the gradiometer system than in the magnetometer system. The increase in signal is determined by multiplying the magnetization of each particle, given by the Langevin function ${ }^{20}$, by its magnetic moment and integrating over the distribution of particles in the sample. For the particles used in our experiment, measurements showed that the $1.2 \mathrm{mT}$ field resulted in a 3.4 times larger signal. Hence, for identical samples, we find that the gradiometer is 30 (decrease in parasitic background 
response $) \times 0.8$ (loss in coupling efficiency $) \times 3.4$ (increase in signal $) \approx 80$ times more sensitive than the magnetometer.

Finally, we estimate the minimum number of superparamagnetic particles the gradiometer can detect. In the immunoassay technique, the particles ${ }^{21}$, nominally $35 \mathrm{~nm}$ in diameter, are bound to specific targets that are immobilized. The gradiometer detects the logarithmic decay of the flux $\Phi(\mathrm{t})$ as the particles undergo Néel relaxation following the removal of the magnetizing field. We fit this signal to ${ }^{22}$

$$
\Phi(\mathrm{t})=\Phi_{\text {offset }}+\Phi_{\mathrm{s}} \ln \left(1+\tau_{\mathrm{mag}} / \mathrm{t}\right)
$$

where $\tau_{\mathrm{mag}}=1 \mathrm{~s}$ is the magnetization time, $\Phi_{\text {offset }}$ is an artifact of the SQUID electronics, and $\Phi_{\mathrm{s}}$ is the fitted amplitude of the flux detected by the gradiometer. We calibrated the sensitivity by depositing magnetic particles on a carbon-coated transmission electron microscopy (TEM) grid, imaging the grid with TEM, and counting the number of particles per unit area. Then we cut the grid into segments and measured the relaxation signal of each piece with a pulsed $1.2 \mathrm{mT}$ field (Fig. 3 upper trace). Between each measurement, we recorded the background decay with no sample present (Fig. 3 lower trace) to confirm there was no residual magnetic material on the window. We fit each relaxation curve, divided the amplitude $\Phi_{\mathrm{s}}$ by the estimated number of particles on the particular grid segment, and averaged the results. We found the mean relaxation amplitude to be $0.8 \mathrm{n} \Phi_{0}$ per particle. The minimum detectable signal is the uncertainty in the value of $\Phi_{\mathrm{s}}, 2.9 \mu \Phi_{\mathrm{o}}$ for 100 averages. (This uncertainty is due solely to the SQUID noise, since the gradiometer has effectively eliminated the parasitic background.) Dividing by the flux per magnetic particle, we arrive at a detection limit (one standard 
deviation) of about 4000 particles $^{23}$. If particles with a larger magnetic moment could be obtained, the sensitivity would improve proportionally. A factor of two improvement in the sensitivity of the gradiometer could be achieved by coupling the pickup loops to the SQUID with a 3-turn, multilayer transformer ${ }^{24}$.

We thank K. Rodriguez for her assistance with the TEM and Mark Alper for ongoing encouragement. This work was supported by the Director, Office of Science, Office of Basic Energy Sciences, Division of Materials Sciences and Engineering of the U.S. Department of Energy under contract number DE-AC03-76SF00098. 
1 J. P. Wikswo, Jr., IEEE Trans. Appl. Supercond. 5, 74 (1995).

${ }^{2}$ Y. P. Ma, I. M. Thomas, A. Lauder, and J. P. Wikswo, Jr., IEEE Trans. Appl. Supercond. 3, 1941 (1993).

${ }^{3}$ F. Baudenbacher, N. T. Peters, and J. P. Wikswo, Jr., Rev. Sci. Instrum. 73, 1247 (2002).

${ }^{4}$ Y. R. Chemla, H. L. Grossman, T. S. Lee, J. Clarke, M. Adamkiewicz, and B. B. Buchanan, Biophys J. 76, 3323 (1999).

5 Y. R. Chemla, H. L. Grossman, Y. Poon, R. McDermott, R. Stevens, M. D. Alper, and J. Clarke, Proc. Natl. Acad. Sci. 97, 14268 (2000).

${ }^{6}$ W. Weitschies, R. Kötitz, T. Bunte, and L. Trahms, Pharm. Pharmacol. Lett. 7, 1 (1997).

${ }^{7}$ K. Enpuku, T. Minotani, T. Gima, Y. Kuroki, Y. Itoh, M. Yamashita, Y. Katakura, and S. Kuhara, Jpn. J. Appl. Phys., Part 2 38, L1102 (1999).

${ }^{8}$ K. Enpuku, M. Hotta, and A. Nakahodo, Physica C. 357-360, 1462 (2001).

9 H. Weinstock, IEEE Trans. Magn. 27, 3231 (1991).

${ }^{10}$ R. C. Black, F. C. Wellstood, E. Dantsker, A. H. Miklich, J. J. Kingston, D. T. Nemeth, and J. Clarke, Appl. Phys. Lett. 64, 100 (1994).

11 M. Mück, M. v. Kreutzbruck, U. Baby, J. Troell, and C. Heiden, Physica C 282-287, 407 (1997).

12 Y. Watanabe, S. H. Kang, J. W. Chan, J. W. Morris, T. J. Shaw, and J. Clarke, J. Appl. Phys. 89, 1977 (2001). 
13 S. Knappe, D. Drung, T. Schurig, H. Koch, M. Klinger, and J. Hinken, Cryogenics 32, 881 (1992).

14 T. S. Lee, E. Dantsker, and J. Clarke, Rev. Sci. Instrum. 67, 4208 (1996).

15 Bradley J. Roth, Nestor G. Sepulveda, and John P. Wikswo Jr., J. Appl. Phys. 65, 361 (1988).

16 All inductances have been calculated using FastHenry software: M. Kamon, M. J. Tsuk, and J. White, IEEE Trans. Microwave Theor. Tech. 42, 1750 (1994).

17 E. Dantsker, S. Tanaka, and J. Clarke, Appl. Phys. Lett. 70, 2037 (1997).

18 R. H. Koch, J. Clarke, W. M. Goubau, J. M. Martinis, C. M. Pegrum, and D. J. van Harlingen, J. Low Temp. Phys. 51, 207 (1983).

19 This area is smaller than the geometrical effective area of the SQUID due to screening by the outer loop.

20 J. S. Blakemore, Solid State Physics 2nd ed. (Cambridge University Press, New York, 1985).

21 Quantum Magnetics, Madison, CT.

22 D. V. Berkov and R. Kötitz, J. Phys. Condens. Matter 8, 1257 (1996).

${ }^{23}$ In the paper describing the magnetometer experiment ${ }^{5}$, poor TEM image quality, which we have since improved, may have caused undercounting of the particles. Therefore, the detection limit of the magnetometer, stated as 50,000 particles in the paper, might actually have been higher. This means that our current detection limit of 4000 particles is an improvement of at least a factor of 12 , and likely much greater, over our previous experiments. 
${ }^{24}$ W. Eidelloth, B. Oh, R. P. Robertazzi, W. J. Gallagher, and R. H. Koch, Appl. Phys. Lett. 59, 3473 (1991).

Fig. 1 Upper trace: magnetic background detected by the magnetometer in response to a $0.3 \mathrm{mT}$ field, fitted to $\Phi(\mathrm{t})=\Phi_{\text {offset }}+\Phi_{\mathrm{s}} \mathrm{e}^{-\mathrm{t} / \tau}$ (dotted line); lower trace: background detected by the gradiometer in response to a $1.2 \mathrm{mT}$ field. Applied field was pulsed on for $1 \mathrm{~s}$ and off for $1 \mathrm{~s}$, and data were recorded each time the field was turned off; 100 averages were taken. Inset: Configuration of the SQUID microscope. The sample, at room temperature and atmospheric pressure, is $100 \mu \mathrm{m}$ above the SQUID, which is at $77 \mathrm{~K}$ and in a vacuum.

Fig. 2 Schematic of the gradiometer. Lower loop attached to the SQUID reduces imbalance caused by the SQUID.

Fig. 3 Upper trace: signal from superparamagnetic nanoparticles deposited on a TEM grid versus time fitted to $\Phi(t)=\Phi_{\text {offset }}+\Phi_{\mathrm{s}} \ln \left(1+\tau_{\mathrm{mag}} / \mathrm{t}\right)$ (solid line $)$; lower trace: magnetic background with no sample present, fitted to $\Phi(t)=\Phi_{\text {offset }}+\Phi_{s} e^{-t / \tau}$ (solid line). A $1.2 \mathrm{mT}$ field was pulsed on for $1 \mathrm{~s}$ and off for $1 \mathrm{~s}$, and data were recorded each time the field was turned off; 100 averages were taken. Inset: Néel relaxation amplitude versus gradiometer-sample separation. Dots represent experimental data, curve was calculated from our model and scaled to fit the data. 


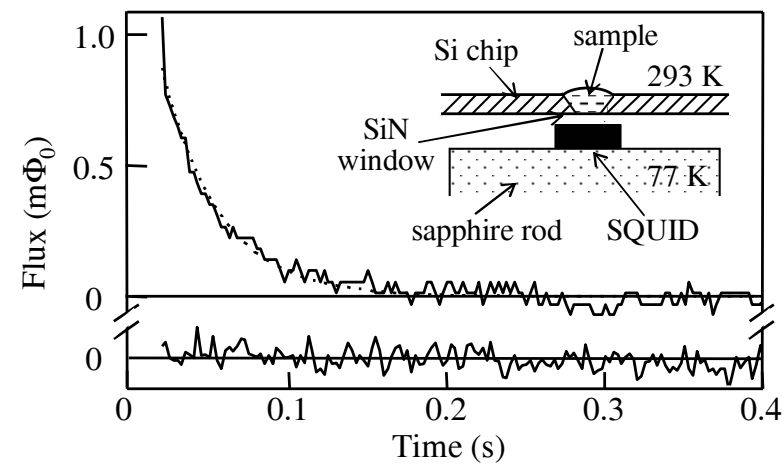




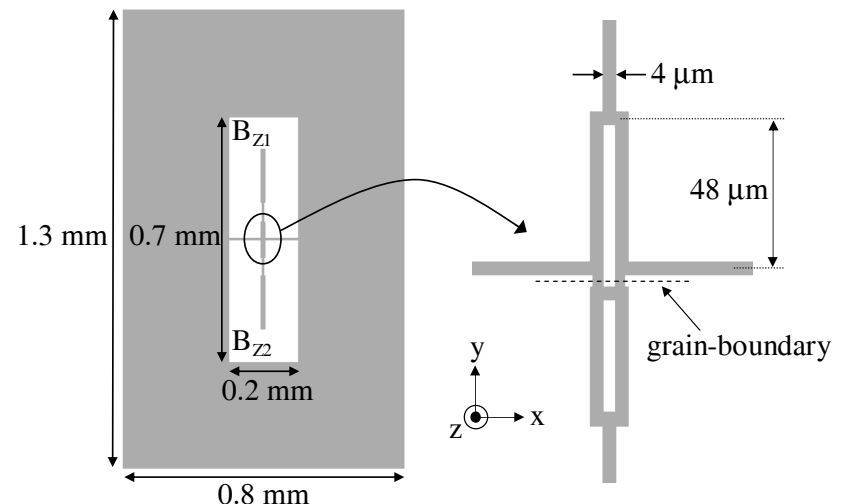




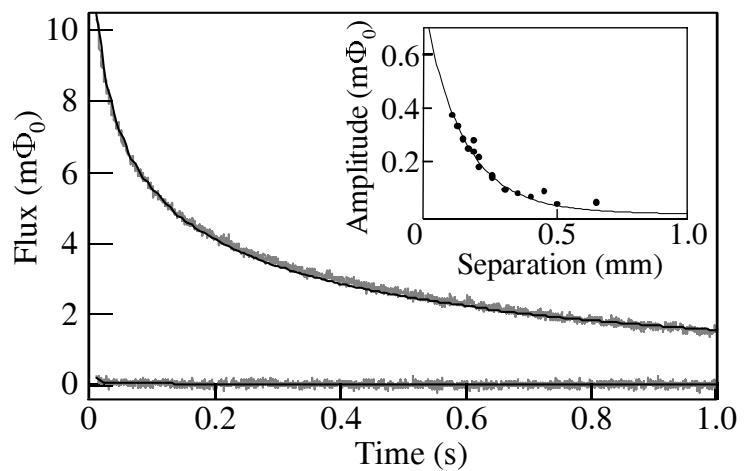

